\title{
Oxidation-Induced Surface Plasmon Band Fragmentation in Silver Clusters
}

\author{
Romain Schira and Franck Rabilloud* \\ Université de Lyon, F-69003, Lyon, France; Université Lyon 1, Villeurbanne; UMR5306, \\ Institut Lumière Matière
}

E-mail: franck.rabilloud@univ-lyon1.fr 


\begin{abstract}
The optical absorption of silver clusters is characterized by a strong plasmon band. However, most of the experimental photoabsorption measurements are performed on clusters interacting with a surrounding media, which can induce oxidation processes and possibly modify the optical properties. Here, we investigate the effects of oxygen adsorption onto the electronic structure and the optical properties of silver $\operatorname{Ag}_{n}$ ( $n=8,20,38)$ clusters by using a well-established methodology based on the TimeDependent Density Functional Theory (TDDFT). The presence of oxygen atoms on the silver cluster is found to induce a fragmentation of the plasmon resonance that can lead to its disappearance and the onset of many excitations having a low plasmonic character. The broadening of the plasmon band is shown to depend on the number of oxygen atoms but also on their positions with respect to the cluster. The interaction of $\mathrm{Ag}_{8}$ clusters with molecular $\mathrm{O}_{2}$ is also examined. Our study can be used to analyze experimental measurements related to oxygen chemisorption or physisorption on metal clusters, and can also be considered as a first-step to model optical properties of noble metal nanoclusters embedded in oxide matrices.
\end{abstract}

\title{
Introduction
}

Metallic nanoclusters has been a matter of considerable study for several decades notably because of their unique electronic and optical properties. ${ }^{1,2}$ In particular, the Localized Surface-Plasmon Resonance (LSPR) phenomenon, characterized by a very intense absorption band, can be observed on those systems. The LSPR, described as a collective excitation of conduction electrons due to an incident electric field, makes metallic clusters suitable or promising for a wide range of applications, such as photothermal therapy, ${ }^{3,4}$ biological sensing, ${ }^{5}$ photocatalysis, ${ }^{6}$ Surface-Enhanced Raman Spectroscopy (SERS), ${ }^{7,8}$ photovoltaics, ${ }^{9,10}$ etc.

The LSPR frequency depends on the size, shape, composition ${ }^{11,12}$ and environment ${ }^{13,14}$ 
of the clusters. Even if the LSPR can be investigated on clusters in gas phase ${ }^{15}$ most of the experimental photoabsorption measurements are performed on clusters interacting with a surrounding media, i.e., deposited on a surface, ${ }^{16,17}$ suspended in a solution, ${ }^{18}$ or embedded in solid matrix. ${ }^{14,19}$ Trapping allows to obtain a high density and to control the stability of clusters, which facilitates experimental measurements. However, the interactions between the surrounding media and the clusters are complex and generally poorly understood, which makes the analysis of experimental results difficult. ${ }^{14}$ Several spectroscopic data obtained on nanometric silver clusters embedded in glass, ${ }^{20}$ alumina ${ }^{21,22}$ and silica ${ }^{14,16,23}$ matrices are referenced in the literature. Globally, oxide matrices cause a red-shift of the LSPR frequency and a broadening of the bandwidth compared to results obtained in gas phase. ${ }^{13,16}$ Both the red-shift and the broadening depend on the matrix composition and porosity, and can be described with empirical, semi-classical methods for clusters containing at least a thousand of atoms. ${ }^{24}$ Oxide matrices may also induce the formation of Ag-O bonds, a deformation of the cluster, oxidation, and a valence electron confinement. A precise description of those effects, taking into account the details of the atomic structure, is required to trace back the intrinsic properties of the metal cluster. A first-step to correctly model the effects of oxide matrices on a silver cluster is to study the interactions of the cluster with oxygen.

Experimentally, the interaction of oxygen with silver clusters in the gas phase has motived many studies. ${ }^{25,26}$ At $77 \mathrm{~K}$ molecular oxygen was found to stick chemisorbed to small $\mathrm{Ag}_{n}$ clusters $(n<70)$ with a transfer of one electron, while above $105 \mathrm{~K}$ the chemisorption transforms into oxidation, invoking the dissociation of the oxygen molecule. ${ }^{25}$ Various theoretical studies using the Density Functional Theory (DFT) focused on the geometrical structure of small oxidized silver. The lowest isomers of charged and neutral $\operatorname{Ag}_{n} \mathrm{O}_{m}$, with $n$ $=1-6$, and $m=1-2$ have been determined with a Local Spin Density Approximation (LSDA) exchange-correlation functional. ${ }^{27}$ Geometrical structures of clusters containing less than 9 silver atoms in interaction with molecular and dissociated $\mathrm{O}_{2}$ have been investigated using a Generalized Gradient Approximation (GGA) functional. ${ }^{28}$ It appears from those works that 
the oxygen atoms can induce important structural deformations in small clusters, and in most cases a dissociative $\mathrm{O}_{2}$ adsorption is preferred. A more general study of the geometrical structure of neutral and anionic silver clusters containing up to 25 atoms and interacting with molecular oxygen have been realized with GGA and hybrid density-functionals. ${ }^{29}$ This study reveals the fact that the binding energy of oxygen atoms and molecular oxygen, as well as ionization potentials and electron affinities follow an odd/even oscillation as a function of cluster size. Absorption spectra of $\mathrm{Ag}_{3} \mathrm{O}$ and $\mathrm{Ag}_{4} \mathrm{O}$, and the one of different isomers of $\mathrm{Ag}_{4} \mathrm{O}, \mathrm{Ag}_{10} \mathrm{O}_{4}$ and $\mathrm{Ag}_{10} \mathrm{O}_{5}$, have been computed using the equation-of-motion coupledcluster (EOM-CC) ${ }^{30}$ and Random Phase Approximation (RPA) ${ }^{31}$ methods. The authors conclude that the $p$-electrons of oxygen atom activate the $d$-electrons of silver, leading to important changes in the optical response.

None of the previous theoretical works has focused on the effects induced by oxidation on the plasmon resonance in silver clusters. It is well known that the plasmon-like band in silver is clearly visible down to small-size bare clusters of about 18-20 atoms. ${ }^{19}$

To investigate the role of oxidation on the optical response of silver clusters, and especially on the LSPR phenomenon, we examined here the absorption spectra of pure and oxidized $\mathrm{Ag}_{8}, \mathrm{Ag}_{20}$ and $\mathrm{Ag}_{38}$ in the framework of the Time-Dependent DFT (TDDFT). ${ }^{32}$ This method gives precise results on silver clusters provided that an appropriate exchangecorrelation functional is used. ${ }^{19,33-35}$ For silver clusters, the description of excited states in TDDFT within the adiabatic approximation requires a correction of the self-interaction error (SIE) and a correct asymptotic behaviour. The best results ${ }^{36}$ have been obtained with range separated hybrid functionals (RSHs). Those functionals significantly reduce the SIE problems and improve the asymptotic behaviour at long range thanks to the inclusion of Hartree-Fock exchange. ${ }^{37,38}$ The present paper is organized as follows: in section 2 , we present our approach to generate the geometrical structure of $A g_{m} O_{n}(m=8,20,38$, and $n=1,2,3)$ and to compute their optical properties. In section 3, we discuss the electronic structure and absorption spectra and we analyze the excited states of pure and oxidized 
clusters. This section will finish with a discussion of the effects induced by the dioxygen $\mathrm{O}_{2}$ onto the optical response of $\mathrm{Ag}_{8}$.

\section{Computational Method}

DFT and TDDFT calculations presented in this paper have been performed with the RSH exchange-correlation functional $\omega \mathrm{B} 97 \mathrm{x},{ }^{39}$ which includes an increasing part of exact HartreeFock exchange at long range (from $16 \%$ at short range to $100 \%$ at long range). Silver atoms were described with a relativistic effective core potential (RECP), so that only 19 valence electrons per silver atom were treated explicitly $\left(4 s^{2}, 4 p^{6}, 4 d^{10}, 5 s^{1}\right)$, together with the corresponding Gaussian basis set LanL2DZ. ${ }^{40}$ The use of this functional and basis set leads to a precise description of the absorption spectra of pure silver clusters and a suitable description on the plasmon phenomenon. ${ }^{19,36,41}$ The oxygen atoms have been modelled with the 6 -31G* basis set. ${ }^{42}$ For molecular oxygen, the use of this basis set leads to a binding energy and bond length of respectively $5.43 \mathrm{eV}$ and $1.20 \AA$, in good agreement with the experimental ones, referenced as $5.23 \mathrm{eV}$ and $1.21 \AA .^{28}$

The geometrical structures of $\mathrm{Ag}_{8}$ and $\mathrm{Ag}_{38}$ were taken from a study by Chen et $\mathrm{al}^{43}$ dedicated to the prediction of structures of silver clusters using a genetic algorithm with an embedded atom method potential. Let us note that for $\mathrm{Ag}_{8}$ we have been considering the two lowest isomers found by Chen et al, respectively of symmetry $T_{d}$ and $D_{2 d}$, as both of them likely coexist in experimental samples. ${ }^{41}$ For the geometrical structure of $\mathrm{Ag}_{20}$, we considered the ground state structure of $C_{s}$ symmetry optimized at DFT level, ${ }^{44}$ this isomer gives an absorption spectra very close to the experimental one. ${ }^{19}$ All the geometrical structures were relaxed with $\omega \mathrm{B} 97 \mathrm{x}$.

To determine the lowest-energy isomer of the oxidized clusters $\operatorname{Ag}_{m} \mathrm{O}_{n}(m=8,20,38$, and 
$n=1,2,3)$, we randomly placed oxygen atoms around the pure silver clusters. For $\mathrm{Ag}_{8} \mathrm{O}_{n}$, 80 structures have been generated for each value of $n$; for half of them, the starting point was $\mathrm{Ag}_{8}$ in the $T_{d}$ symmetry, and for the second half it was $\mathrm{Ag}_{8}$ in the $D_{2 d}$ symmetry. For $\mathrm{Ag}_{20} \mathrm{O}_{n}$ and $\mathrm{Ag}_{38} \mathrm{O}_{n}$, up to 80 structures have been generated for each value of $n$. Then, we relaxed all the structures with $\omega \mathrm{B} 97 \mathrm{x}$ and we selected only the lowest-energy isomer for each $\mathrm{Ag}_{m} \mathrm{O}_{n}$ systems for the present study. These are all singlet. Let us note that optimizations of $\mathrm{Ag}_{8} \mathrm{O}_{n}$ that start from $\mathrm{Ag}_{8}$ with a $T_{d}$ or $D_{2 d}$ symmetry lead to the same lowest-energy isomer.

All DFT and TDDFT calculations were performed using the program Gaussian09. ${ }^{45}$ Preand post-processing operations were performed by using the graphical interface Gabedit. ${ }^{46}$

\section{Results and discussion}

The optimized structures of $\mathrm{Ag}_{8}, \mathrm{Ag}_{20}, \mathrm{Ag}_{38}$ and $\mathrm{Ag}_{m} \mathrm{O}_{n}(m=8,20,38$, and $n=1,2$, 3) are presented in Figure 1. Oxygen atoms preferentially stay on the surface. They are adsorbed in 3 or 4-fold position, except for $\mathrm{Ag}_{8} \mathrm{O}_{3}$ where an oxygen atom is situated between the cluster and a silver atom. The isomer obtained for $\mathrm{Ag}_{8} \mathrm{O}_{2}$ is already referenced in the literature as being the most steady one. ${ }^{28}$ Deformation induced by the presence of $\mathrm{O}$ atoms are significant if the number of oxygen atoms is important or if the number of silver atoms is small. The adsorption of a single oxygen atom on $\mathrm{Ag}_{20}$ leads to a small elongation of the interatomic distances for Ag atoms bound to the oxygen. The presence of two or three oxygen atoms induces a stronger reorganization in surface while two Ag atoms stay in the core. The adsorption of one or two oxygen atoms on $\mathrm{Ag}_{38}$ only induces minor changes in the frame of silver clusters, but a stronger deformation is observed at the surface when three $\mathrm{O}$ atoms are adsorbed.

The minimal distance between oxygen and silver atoms, as well as the averaged charge 


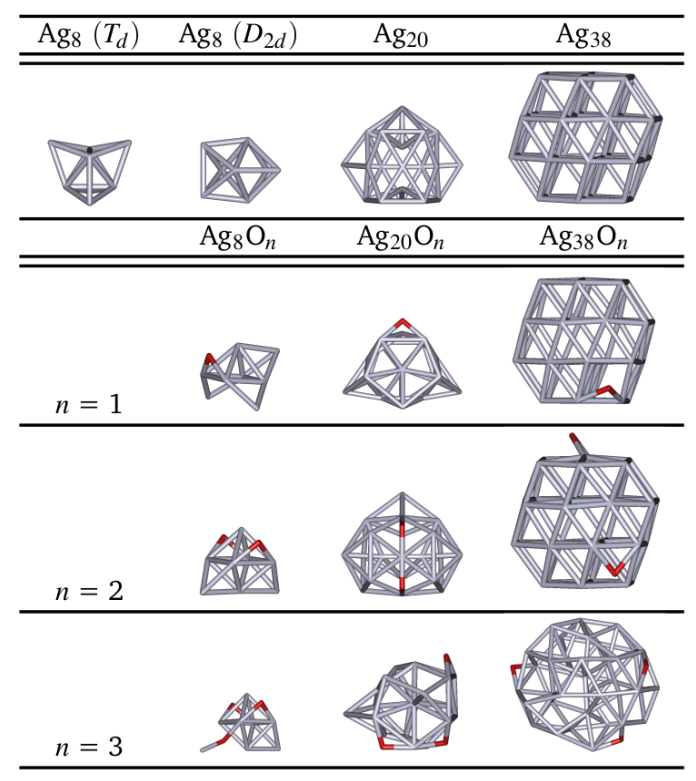

Figure 1: Geometrical structures of pure $\left(\mathrm{Ag}_{8}\right.$ with $T_{d}$ and with $D_{2 d}$ symmetry, $\mathrm{Ag}_{20}$, and $\left.\mathrm{Ag}_{38}\right)$ and oxidized $\left(\mathrm{Ag}_{m} \mathrm{O}_{n}, m=8,20,38\right.$, and $\left.n=1,2,3\right)$ silver clusters. The oxygen atoms are represented in red.

and the binding energy of oxygen atoms are referenced in the Table 1 . The minimal distance between oxygen and silver atoms varies between 2.09 and $2.24 \AA$, which is close to the value obtained with LSDA calculation on small oxidized silver clusters. ${ }^{27}$ The charges indicate that all oxygen atoms attract at least one electron. Let us note that the present binding energy obtained for $\mathrm{Ag}_{8} \mathrm{O}_{2}$ is in qualitative agreement with the referenced one. ${ }^{28}$

The absorption spectra of the pure and oxidized silver clusters are presented in Figure 2. The oxygen atoms have an important effect on the optical response of clusters. Absorption of pure silver clusters are dominated by a few intense transitions close to $4.0 \mathrm{eV}$. When oxygen atoms are present, the few intense transitions are replaced by many transitions having a low oscillator strength and spread over a wide range of energy. A single oxygen atom is enough to produce a significant change in the optical response of $\mathrm{Ag}_{8}$. The absorption of $\mathrm{Ag}_{8}$ with symmetry $T_{d}$ is mainly due to three degenerated transitions situated at $4.04 \mathrm{eV}$, while the spectrum of the $D_{2 d}$-symmetry structure is dominated by transitions located at 3.74 and $4.00 \mathrm{eV}$. By contrast, the spectrum of $\mathrm{Ag}_{8} \mathrm{O}$ contains many transitions and none 
Table 1: Minimal distance between $\mathrm{Ag}$ and $\mathrm{O}$ atoms ( $\mathrm{D}_{\min }$, in $\AA$ ), averaged charge over $\mathrm{O}$ atoms $(\mathrm{q}(\mathrm{O})$, in $|e|)$, and averaged binding energy of $\mathrm{O}$ atoms $\left(\mathrm{E}_{b}\right.$, en $\mathrm{eV})$ in oxidized silver clusters $\operatorname{Ag}_{m} \mathrm{O}_{n}(m=8,20,38$, and $n=1,2,3)$. The atomic charge is estimated through the natural population analysis (NPA). ${ }^{47}$

\begin{tabular}{ccccc}
\hline $\mathrm{Ag}_{m} \mathrm{O}_{n}$ & & $m=8$ & $m=20$ & $m=38$ \\
\hline \hline \multirow{4}{*}{$n=1$} & $\mathrm{D}_{\min }$ & 2.14 & 2.16 & 2.24 \\
& $\mathrm{q}(\mathrm{O})$ & -1.12 & -1.13 & -1.22 \\
& $\mathrm{E}_{b}$ & 3.07 & 3.36 & 4.11 \\
\hline \multirow{4}{*}{$n=2$} & $\mathrm{D}_{\min }$ & 2.14 & 2.14 & 2.18 \\
& $\mathrm{q}(\mathrm{O})$ & -1.06 & -1.14 & -1.17 \\
& $\mathrm{E}_{b}$ & 3.37 & 3.48 & 3.68 \\
\hline \multirow{4}{*}{$n=3$} & $\mathrm{D}_{\min }$ & 2.05 & 2.09 & 2.12 \\
& $\mathrm{q}(\mathrm{O})$ & -1.09 & -1.13 & -1.18 \\
& $\mathrm{E}_{b}$ & 3.26 & 3.35 & 3.98 \\
\hline
\end{tabular}

of them can easily be associated to the main one of the pure $\mathrm{Ag}_{8}$ clusters. The changes in the global appearance induced by a single oxygen atom on the spectra of $\mathrm{Ag}_{20}$ and $\mathrm{Ag}_{38}$ are not as important as in the case of $\mathrm{Ag}_{8}$. The oxygen atom gives rise to many transitions having a non-negligible intensity, but spectra of $\mathrm{Ag}_{20} \mathrm{O}$ and $\mathrm{Ag}_{38} \mathrm{O}$ are still dominated by an absorption band situated around $4.0 \mathrm{eV}$. However, the width of the main absorption band increases when oxygen atoms are added, and the spectrum of $\mathrm{Ag}_{20} \mathrm{O}_{3}\left(\mathrm{Ag}_{38} \mathrm{O}_{3}\right)$ strongly differs from the one of pure $\mathrm{Ag}_{20}$ (respectively $\mathrm{Ag}_{38}$ ) cluster.

It is known that $\mathrm{Ag}_{20}$ and $\mathrm{Ag}_{38}$ have a plasmonic response in gas phase. ${ }^{36}$ As suggested by Figure 2, oxidation leads to the decline of the plasmonic resonance on those clusters. This statement is also supported by experimental measurements. Lünskens et al ${ }^{48}$ have used Second Harmonic Generation Spectroscopy (s-SHG) to study the effect of oxidation on supported silver clusters. They reported a significant decrease of s-SHG signal when clusters containing at least twenty silver atoms are exposed to oxygen. The spectra obtained by Lünskens et. al. on oxidized clusters are dominated by a wide absorption band and have similar shapes than the ones we calculated. A theoretical study using the Time-Dependent Density Functional Tight Binding method over aluminium nanoclusters also shows that oxidation lead to 
an important decrease of plasmon resonance intensity, ${ }^{49}$ which is in quantitative agreement with what we obtained with silver.
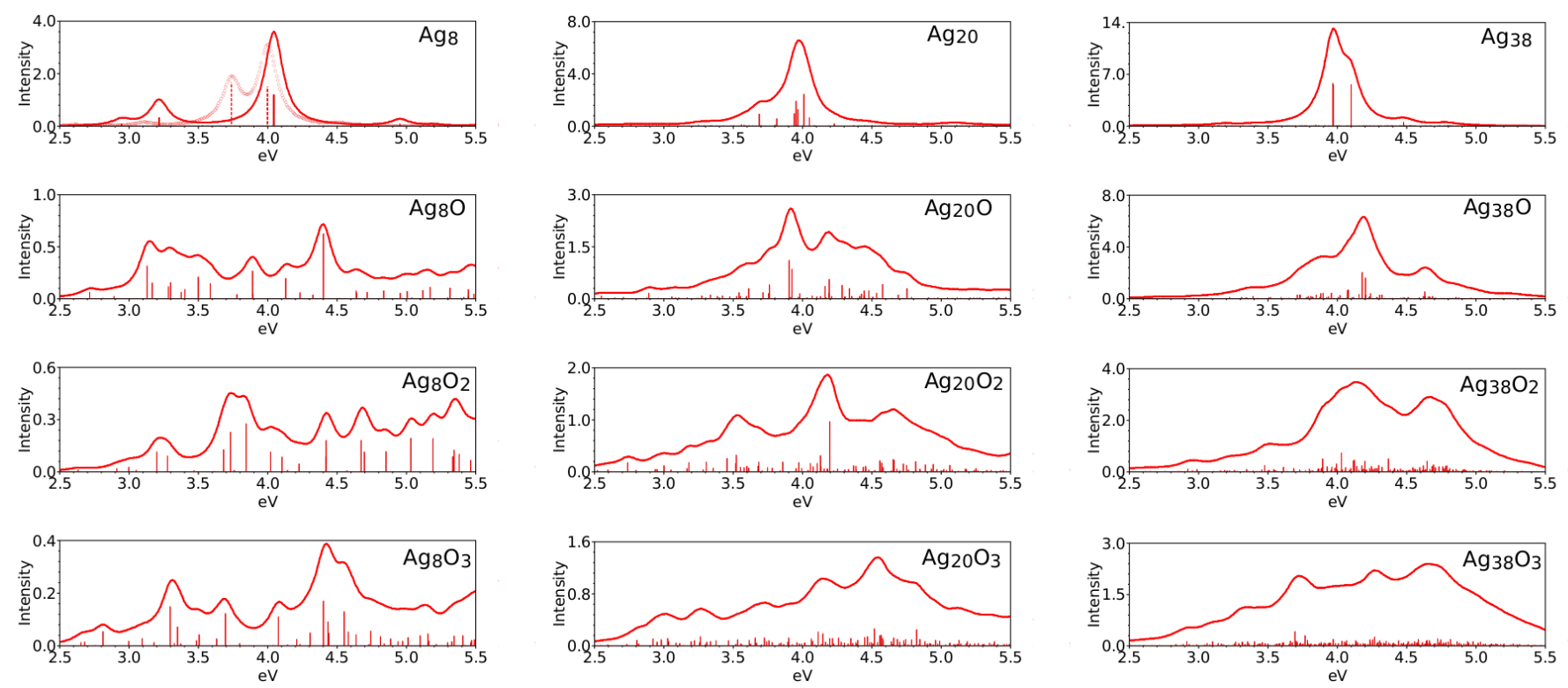

Figure 2: Absorption spectra of $\mathrm{Ag}_{8}, \mathrm{Ag}_{20}, \mathrm{Ag}_{38}$ (first row) and $\mathrm{Ag}_{m} \mathrm{O}_{n}(m=8,20,38$, and $n=1,2,3)$. Spectra are plotted with a Lorentzian broadening $(\mathrm{FWHM}=0.08 \mathrm{eV})$. For $\mathrm{Ag}_{8}$ the solid lines (transitions and convolution) correspond to the spectrum of the $T_{d}$-symmetry isomer and the others (dashed transition and dotted convolution) to the spectrum of the $D_{2 d}$-symmetry structure.

The density of states of pure $\mathrm{Ag}_{20}$ and $\mathrm{Ag}_{38}$ silver clusters, as well as the one of $\mathrm{Ag}_{20} \mathrm{O}$, $\mathrm{Ag}_{20} \mathrm{O}_{2}, \mathrm{Ag}_{38} \mathrm{O}$ and $\mathrm{Ag}_{38} \mathrm{O}_{2}$ are depicted in Figure 3. Some of the calculated Kohn-Sham orbitals are also represented in this figure. Our description of the density of states for oxidized silver cluster is in quantitative agreement with a previous DFT study concerning the oxidation of $\mathrm{Ag}_{6} \cdot{ }^{28}$ It is well established that the electronic structure of silver clusters are in agreement with the shell model ${ }^{19,50}$ in which the $s$ valence electrons are distributed in delocalized orbitals with the following energy sequence : $1 \mathrm{~S}^{2}, 1 \mathrm{P}^{6}, 1 \mathrm{D}^{10}, 2 \mathrm{~S}^{2}, 1 \mathrm{~F}^{14}, 2 \mathrm{P}^{6}$, $1 \mathrm{G}^{18}$, etc. One can easily identify Kohn-Sham orbitals associated to different shells for pure $\mathrm{Ag}_{20}$ and $\mathrm{Ag}_{38}$. The electronic structure of $\mathrm{Ag}_{20}$ ends with a full $2 \mathrm{~S}$ shell, and the one of $\mathrm{Ag}_{38}$ ends with an open $2 \mathrm{P}$ shell. For the oxidized cluster, the $2 p$ orbital of the oxygen, that we will note $\mathrm{P}_{O x}$, appears in the valence band of the silver cluster, leading to hybridization 

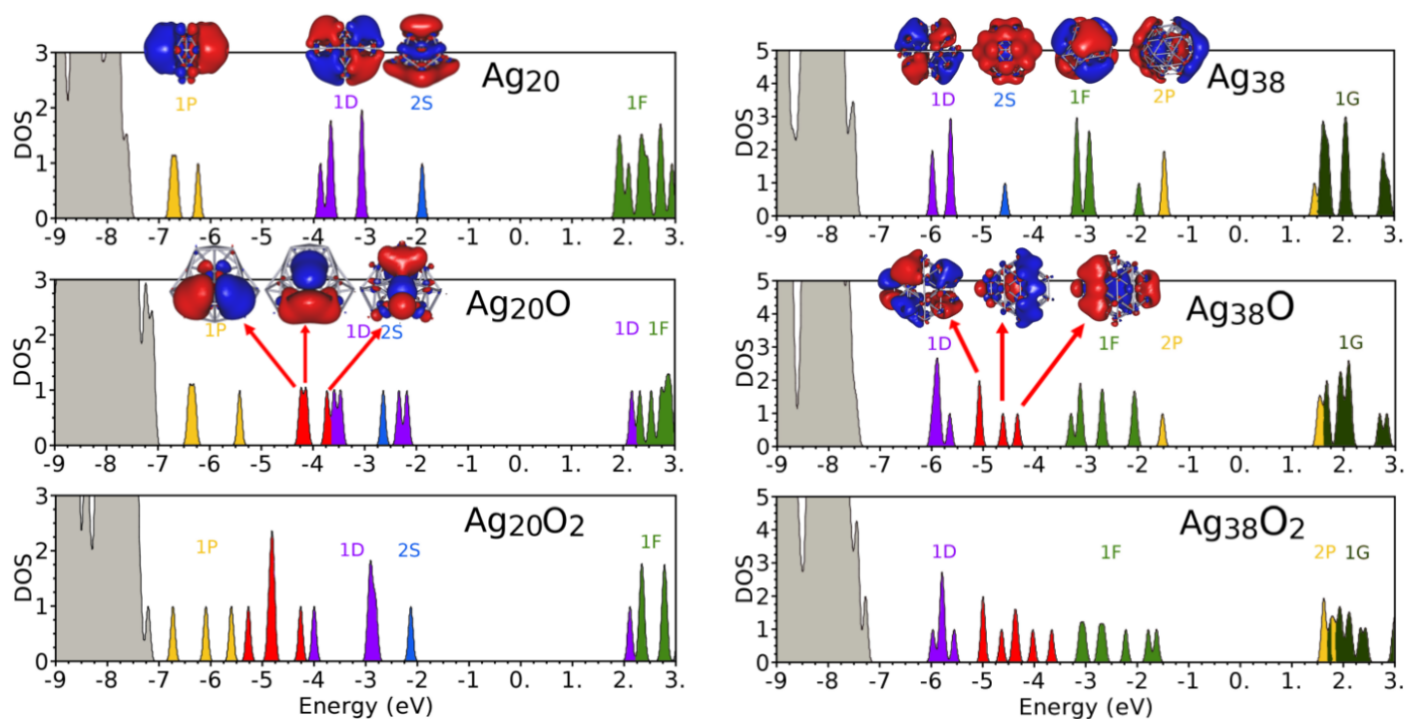

Figure 3: Density of states of $\mathrm{Ag}_{20}, \mathrm{Ag}_{20} \mathrm{O}, \mathrm{Ag}_{20} \mathrm{O}_{2}, \mathrm{Ag}_{38}, \mathrm{Ag}_{38} \mathrm{O}, \mathrm{Ag}_{38} \mathrm{O}_{2}$. The colours correspond to the different types of molecular orbitals, and the grey band represent the $d$ electrons occupation. The molecular orbitals of type $1 \mathrm{P}, 1 \mathrm{D}$ and $2 \mathrm{~S}$ for $\mathrm{Ag}_{20}$ as well as the $1 \mathrm{D}, 2 \mathrm{~S}, 1 \mathrm{~F}, 2 \mathrm{P}$ of $\mathrm{Ag}_{38}$ are drawn. The states having an important component over the oxygen atoms are in red, and the associated orbitals are represented for $\mathrm{Ag}_{20} \mathrm{O}$ and $\mathrm{Ag}_{38} \mathrm{O}$.

of those orbitals. In Figure 3, states that have a strong $\mathrm{P}_{O x}$ character are represented in red and the corresponding orbitals are shown for $\mathrm{Ag}_{20} \mathrm{O}$ and $\mathrm{Ag}_{38} \mathrm{O}$. Since oxygen atoms attract electrons, some valence states of silver that are occupied in the case of pure silver cluster move to the conduction band when oxygen atoms are adsorbed. For $\mathrm{Ag}_{20}$, some of the 1D orbitals become virtual when oxygen is added, and the adsorption of two oxygen atoms empty the whole $2 \mathrm{P}$ band of $\mathrm{Ag}_{38}$. Hence, the presence of $\mathrm{P}_{O x}$ orbitals in the valence band, with the subsequent hybridization between silver and oxygen orbitals, as well as the presence of new virtual states, leads to new optically allowed transitions which were not observed in pure metal clusters. Consequently, the absorption band is significantly broadened.

Oxidation induces a broadening and a damping of the plasmon band. The few intense transitions computed in the case of pure silver clusters are fragmented into many peaks which are scattered across a large range of frequencies when oxygen atoms are adsorbed. Figure 4 and Figure 5 show that this fragmentation is associated to the loss of the plas- 


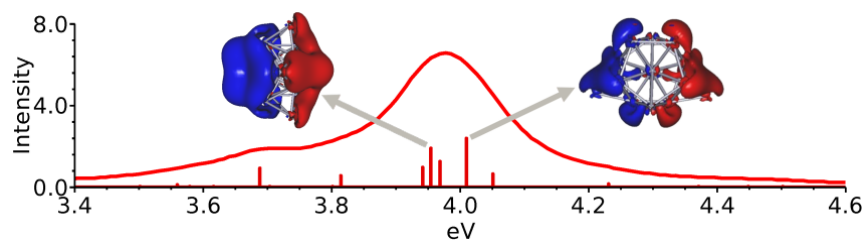

Figure 4: Absorption spectra of $\mathrm{Ag}_{20}$. The isosurface plots of the transition density for two main peaks of $\mathrm{Ag}_{20}$, situated at 3.95 and $4.01 \mathrm{eV}$, are represented. The red and blue surfaces respectively indicate positive and negative isovalues of \pm 0.0003 .

monic character of the excitations. A simple way to investigate the plasmonic character of an electronic transition is to plot its transition density. ${ }^{36}$ The absorption spectra and the isosurface plot of the transition density of the two most intense excitations of $\mathrm{Ag}_{20}$ situated inside the main absorption band are given in Figure 4. The transition densities display a typical dipolar shape, which represents the oscillation of the electron density along the direction of the perturbative electric field. This type of signature is typical of excitation having an important plasmonic character. ${ }^{36}$ Plots of the transition density of the five most intense peaks belonging to the main absorption band of $\mathrm{Ag}_{20} \mathrm{O}$ and $\mathrm{Ag}_{20} \mathrm{O}_{2}$ are represented in Figure 5. They reveal many alternations of positive and negative zones. The alternations of sign are more numerous for $\mathrm{Ag}_{20} \mathrm{O}_{2}$ than for $\mathrm{Ag}_{20} \mathrm{O}$. For oxidized clusters, the features of the density transition differ significantly from the ones found on the pure $\mathrm{Ag}_{20}$ cluster. This show that excitation computed on $\mathrm{Ag}_{20} \mathrm{O}$ and $\mathrm{Ag}_{20} \mathrm{O}_{2}$ do not have a strong plasmonic character. Thus the presence of oxygen atoms on the silver cluster induces a fragmentation of the plasmon resonance and replaces it by many excitations having a low plasmonic character.

Interestingly, the position of the oxygen atom with respect to the silver cluster has a non negligible effect over the optical properties. We built a structure of $\operatorname{Ag}_{20} \mathrm{O}$, hereafter noted $\mathrm{Ag}_{20} \mathrm{O}_{\text {inside }}$, where the oxygen is encapsulated inside the silver cluster. To do so, we replaced the only silver atom of $\mathrm{Ag}_{20}$ which is not at the surface with an oxygen atom and we added a silver atom at a random position on the cluster's surface. Then the geometrical structure has been relaxed with the $\omega \mathrm{B} 97 \mathrm{x}$ functional. The structure thereby obtained for $\mathrm{Ag}_{20} \mathrm{O}_{\text {inside }}$, 

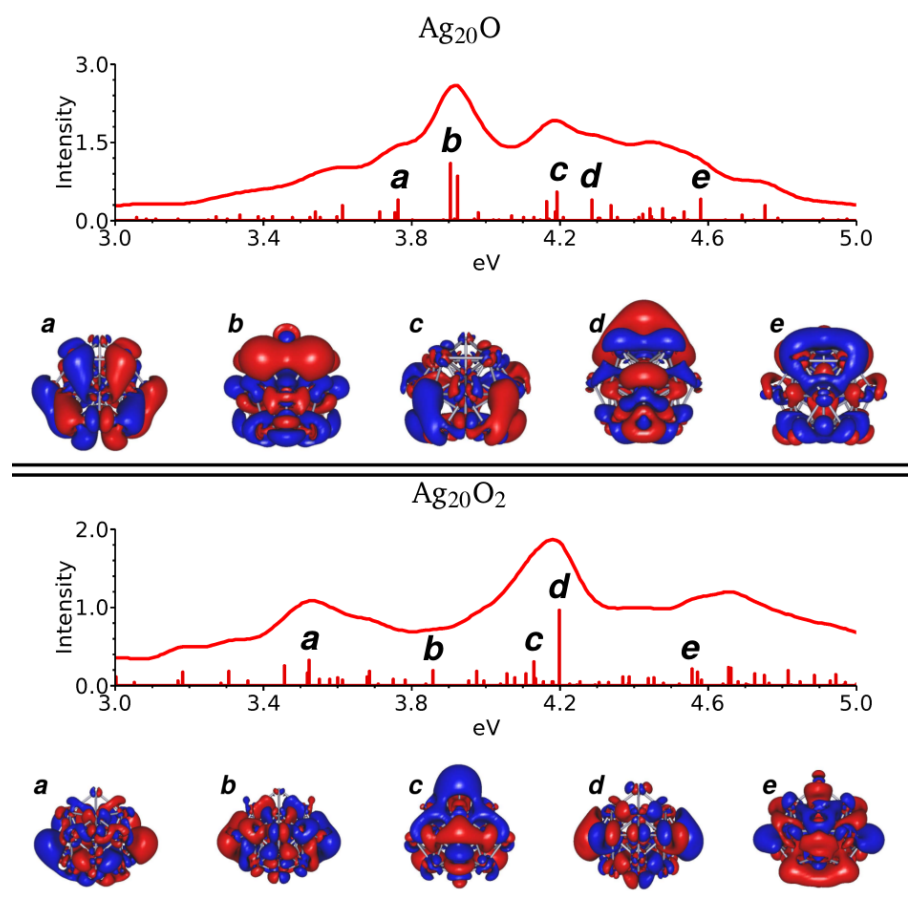

Figure 5: Absorption spectra of $\mathrm{Ag}_{20} \mathrm{O}$ (top) and $\mathrm{Ag}_{20} \mathrm{O}_{2}$ (bottom). Isosurface plots of the transition density (isovalue $= \pm 0.0003$ ) of intense excitations (labeled $a, b, c, d, e$ ) are given below the spectra. Energy of represented transitions for $\mathrm{Ag}_{20} \mathrm{O}$ are 3.76 (a), 3.90 (b), 4.19 (c), $4.29(\mathrm{~d})$, and 4.58 (e) eV, those of $\mathrm{Ag}_{20} \mathrm{O}_{2}$ are 3.52 (a), 3.85 (b), 4.13 (c), 4.19 (d), and 4.57 (e) eV.

which is presented in Figure 6, lies $0.64 \mathrm{eV}$ higher in energy compared to the lowest-energy isomer of $\mathrm{Ag}_{20} \mathrm{O}$. This suggests that oxygen atoms preferably stay at the cluster's surface, which is in agreement with previous DFT study. ${ }^{27,28}$ The electric charge on the oxygen atom placed inside $\mathrm{Ag}_{20}$ is $-1.24|e|$. The density of state and the absorption spectrum of $\mathrm{Ag}_{20} \mathrm{O}_{\text {inside }}$ is given in Figure 6. The ordering of the orbitals is the same than for $\operatorname{Ag}_{20} \mathrm{O}$, shown in Figure 3. The spectra of $\mathrm{Ag}_{20} \mathrm{O}_{\text {inside }}$ and $\mathrm{Ag}_{20} \mathrm{O}$ present some similarities. In both cases many transitions appear in the main absorption band compared to the spectrum of pure $\mathrm{Ag}_{20}$. However, the induced broadening is not as important in the case of $\mathrm{Ag}_{20} \mathrm{O}_{\text {inside }}$ than it is in the case of $\operatorname{Ag}_{20} \mathrm{O}$. When the oxygen atom is inside the silver cluster the intense transitions are all positioned between about 3.8 and $4.2 \mathrm{eV}$. By comparison, when the oxygen atom is adsorbed at the surface, the important transitions lie in an energy range that is at least twice larger. Isosurface plots of the transition density of five peaks of the 
$\mathrm{Ag}_{20} \mathrm{O}_{\text {inside }}$ spectrum are given in the Figure 6. These transition densities do not show as many alternations of sign as in the case of $\mathrm{Ag}_{20} \mathrm{O}$. They are globally distributed in two zones, having opposite sign, on both side of the system, i.e. a somewhat dipolar shape. This type of distribution proofs that, despite of the presence of the oxygen atom, the intense transitions for $\mathrm{Ag}_{20} \mathrm{O}_{\text {inside }}$ have a plasmonic character. In contrast, the presence of $\mathrm{O}$ atoms at the surface prevents the formation of a dipolar mode.

The role the relative position of $\mathrm{O}$ atoms has already been discussed by Burgel et al ${ }^{31}$ when investigating the oxidation of very small silver clusters as $\mathrm{Ag}_{4}$. They concluded that structures with centrally located oxygen atom, and structures where the oxygen is strongly coordinated with multiple neighbours, present a absorption spectrum relatively similar to that of the pure silver cluster. Contrastingly, when the oxygen atom is located in a peripheral position, several new intense peaks spread over a large energy range become visible due to a stronger activation of $d$-electrons of $\mathrm{Ag}$ atoms by $p$-electrons of the $\mathrm{O}$ atom. Our results confirm their statement.

We also studied the interaction between molecular $\mathrm{O}_{2}$ and $\mathrm{Ag}_{8}$ as well as the impact of this molecule on the optical response of this cluster. We focused on $\mathrm{Ag}_{8}$ as it is the smallest cluster we considered in this paper, and so it is likely the most sensitive to the chemical interactions. Experiments on the interaction of gas phases of silver clusters with $\mathrm{O}_{2}$ have shown that molecular $\mathrm{O}_{2}$ can be either physisorbed or chemisorbed on small cationic silver clusters. ${ }^{25,26}$ DFT studies of silver cluster $\mathrm{Ag}_{n}$ in interaction with molecular $\mathrm{O}_{2}$ reveal that dioxygen can be adsorbed at either atop (only one O-Ag bond) or bridge (each $\mathrm{O}$ are bound to the cluster) sites, depending on cluster's size and charge. ${ }^{27-29}$ Those theoretical studies also reveal that the dissociative adsorption (DA) is favored for $n \geq 6$, i.e. the binding energies for molecular adsorption is lower than DA's energies when the silver cluster is large enough to transfer electrons to oxygen atoms. We have considered the attachment of molecular dioxygen on the $T_{d}$-symmetry structure of $\mathrm{Ag}_{8}$. Two types of adsorption were considered: one with the dioxygen in an atop site, and the other one with $\mathrm{O}_{2}$ in a bridge 


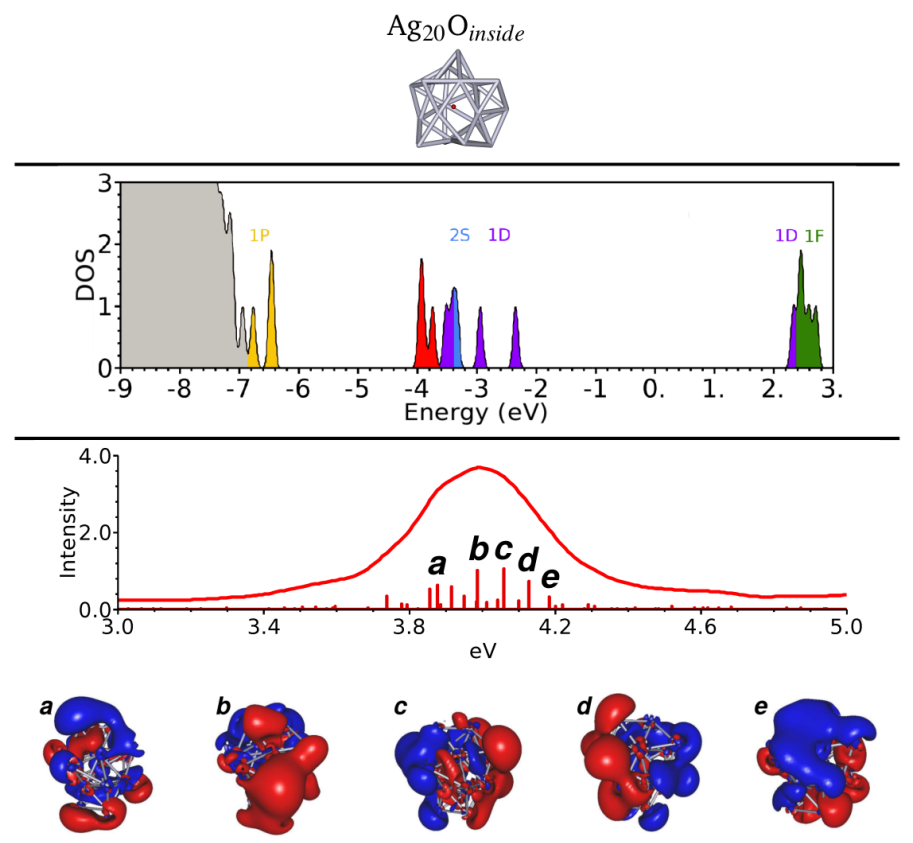

Figure 6: Geometry (top), density of states (middle) of $\mathrm{Ag}_{20} \mathrm{O}_{\text {inside, }}$, together with the absorption spectrum and the density transition (isovalue $= \pm 0.0003$ ) of five intense peaks (bottom). For the density of states, the grey band corresponds to the $d$ electrons, the red states to orbitals that have an important component over the oxygen atom, and others colours to the different shells. The transition densities labeled in the absorption spectra correspond to excitation situated at 3.88 (a), 3.99 (b), 4.06 (c), 4.13 (d) and 4.18 (e) eV.

position. The geometrical structures and the absorption spectra of pure $\mathrm{Ag}_{8}$, and $\mathrm{Ag}_{8}$ in interaction with molecular $\mathrm{O}_{2}$ are given in Figure 7. In all cases, the geometrical configuration obtained with molecular $\mathrm{O}_{2}$ is not as steady as the one obtained with two dissociated oxygen atoms. For $\mathrm{Ag}_{8}$ in interaction with dioxygen on an atop site, the steadiest electronic configuration corresponds to a spin multiplicity of three. For this isomer (Figure 7b), the dioxygen does not induce a strong deformation of the cluster, the binging energy is +0.17 $\mathrm{eV}$, and the $\mathrm{Ag}-\mathrm{O}$ distance is $2.82 \AA$. In the atop position, the oxygen atoms are separated of $1.22 \AA$, i.e. an elongation of only $0.02 \AA$ compared to $\mathrm{O}_{2}$ in gas phase. A singlet state is also found (Figure 7c), it lies $0.15 \mathrm{eV}$ below the dissociation limit of $\mathrm{Ag}_{8}+\mathrm{O}_{2}$ (singlet) and the Ag-O distance is $2.47 \AA$. For $\mathrm{Ag}_{8}$ interacting with dioxygen on a bridge site, the steadiest electronic configuration corresponds to a multiplicity of three (Figure $7 \mathrm{~d}$ ). The configuration lies $0.24 \mathrm{eV}$ below the dissociation limit. The $\mathrm{O}-\mathrm{O}$ bond is elongated to $1.31 \AA$, while 
the shortest $\mathrm{O}-\mathrm{Ag}$ distances are 2.31 and $2.24 \AA$. In the bridge position, the net charge on oxygen atom is $-0.32|e|$ per atom, and that transfer of electron suggests a chemisorption state. The hybridization between the orbitals of the silver cluster and the ones of the dioxygen are important when $\mathrm{O}_{2}$ is in the bridge position, while it is very low for $\mathrm{O}_{2}$ in an atop site.
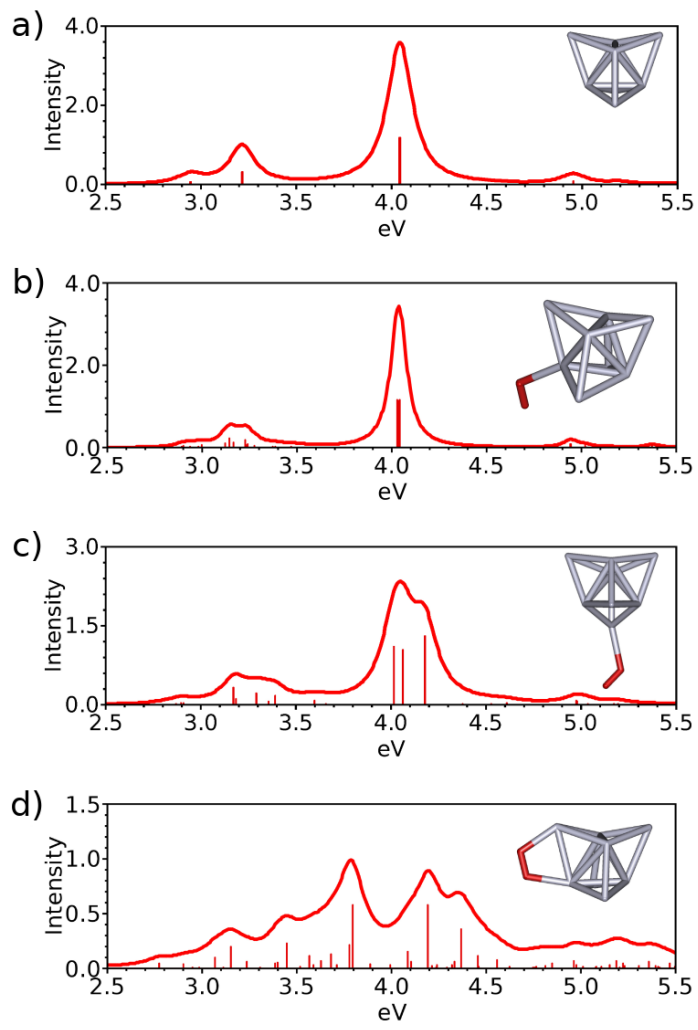

Figure 7: Geometry and absorption spectra of pure $\mathrm{Ag}_{8}$ in the $T_{d}$ symmetry (a). Geometry and absorption spectra of $\mathrm{Ag}_{8}$ in interaction with molecular $\mathrm{O}_{2}$ at the atop position in a triplet state (b), or a singlet state (c), and with $\mathrm{O}_{2}$ in the bridge position (d).

Figure 7 shows that the adsorption of $\mathrm{O}_{2}$ in an atop position does not induce significant changes in the absorption spectra of $\mathrm{Ag}_{8}$. The main transitions of $\mathrm{Ag}_{8}$ in the $T_{d}$ symmetry are three times degenerated and are situated at 3.22, 4.04, and $4.96 \mathrm{eV}$ respectively. With the molecular $\mathrm{O}_{2}$ in an atop position, the system is not in a $T_{d}$ symmetry anymore, and the degeneracy is raised. For the triplet state, $\mathrm{O}_{2}$ is far from silver atoms and the spectrum stay very similar to the one of pure metal cluster (Figure $7 \mathrm{~b}$ ). In the singlet state, the peaks of $\mathrm{Ag}_{8}$ are clearly fragmented, leading to an widening of the bands. The main bands 
are centered at 3.2, 4.1, $5.0 \mathrm{eV}$ respectively (Figure 7c). In both case, the spectrum of $\mathrm{Ag}_{8}$ is quite recognizable. This can be understood by the fact that the molecular $\mathrm{O}_{2}$ is physisorbed in the atop position and thus the orbitals of the silver and oxygen atoms are weakly hybridized. In contrast the absorption spectrum changes significantly (Figure 7d) when the two oxygen atoms bind to the metal cluster. The absorption spectra is dominated by a large band between 3 and $4.5 \mathrm{eV}$, with two main peaks at 3.80 and $4.2 \mathrm{eV}$, followed by a non-negligible tail until $5.5 \mathrm{eV}$. The strong hybridization between silver and oxygen leads to a significant electronic reorganization. Figure 7 reveals that the changes induced by the $\mathrm{O}_{2}$ molecule on the optical response strongly depends on how it is bound to the cluster.

\section{Conclusions}

The optical response of oxidized clusters, containing 8, 20 or 38 silver atoms have been calculated at TDDFT/RSH level. Oxidation is found to induce hybridization and rearrangements in the orbital filling, leading to significant changes on the optical response. Oxygen atoms induce a strong damping and broadening of the absorption band, and can even lead to its disappearance. The broadening of the absorption band is characterized by the fact that the few strong transitions with an important plasmonic character in pure silver clusters are replaced by many transitions spread over a wide range of energy and having low plasmonic character. These new transitions originate from the presence of oxygen orbitals in the valence states, the presence of new virtual states subsequent to charge transfers, and also from hybridization between silver and oxygen orbitals. The damping is all the more important when the number of oxygen atoms is large. However, the effects are reduced when oxygen atom is located inside the metal cluster.

We have also studied the interactions between $\mathrm{Ag}_{8}$ and molecular $\mathrm{O}_{2}$ and demonstrated that changes induced by the molecular adsorption on the absorption spectrum strongly depend on the adsorption mode. Thus spectroscopic measurements seem to be relevant to 
study oxidation process and to discriminate the adsorption modes on metallic clusters.

The present work can be view as a first step to model precisely the effects of an oxide matrice on the optical response of silver clusters. It is worth to emphasize that one should expect a significant damping to the plasmonic response for cluster embedded in those matrices compared to gas phase measurement if chemical bonds are formed between silver and oxygen atoms. From the present study, it can be expected that the adsorption of oxygen atoms on small metal clusters prevents the detection of the plasmon band.

\section{Acknowledgement}

This work was performed using HPC resources from GENCI-IDRIS (Grant A0050807662) and the Pôle Scientifique de Modélisation Numérique (PSMN). It has received a financial support from the French National Research Agency (Agence Nationale de la Recherche, ANR) in the frame of the project FIT SPRINGS, ANR-14-CE08-0009. The authors gratefully acknowledge A.R. Allouche for support for the use of Gabedit. 


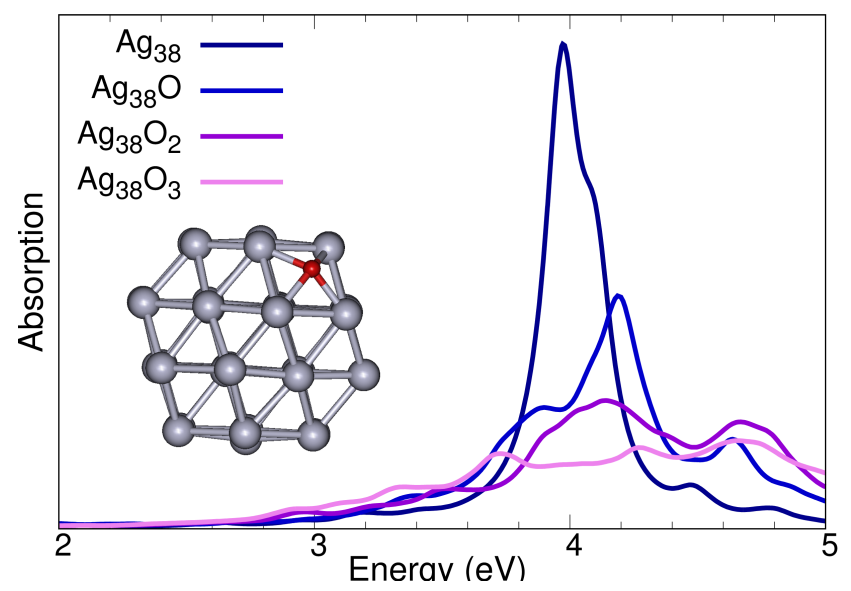

Table of Contents Graphic (TOC) 


\section{References}

(1) Schwartzberg, A. M.; Zhang, J. Z. Novel optical properties and emerging applications of metal nanostructures. The Journal of Physical Chemistry C 2008, 112, 10323-10337.

(2) Mathew, A.; Pradeep, T. Noble metal clusters: applications in energy, environment, and biology. Particle \& Particle Systems Characterization 2014, 31, 1017-1053.

(3) Zhang, J.-Z. Biomedical applications of shape-controlled plasmonic nanostructures: a case study of hollow gold nanospheres for photothermal ablation therapy of cancer. The Journal of Physical Chemistry Letters 2010, 1, 686-695.

(4) Lim, W. Q.; Gao, Z. Plasmonic nanoparticles in biomedicine. Nano Today 2016, 11, $168-188$.

(5) Zeng, S.; Yong, K.-T.; Roy, I.; Dinh, X.-Q.; Yu, X.; Luan, F. A review on functionalized gold nanoparticles for biosensing applications. Plasmonics 2011, 6, 491-506.

(6) Sarina, S.; Waclawik, E. R.; Zhu, H. Photocatalysis on supported gold and silver nanoparticles under ultraviolet and visible light irradiation. Green Chemistry 2013, $15,1814-1833$.

(7) Stamplecoskie, K. G.; Scaiano, J. C.; Tiwari, V. S.; Anis, H. Optimal size of silver nanoparticles for surface-enhanced Raman spectroscopy. The Journal of Physical Chemistry C 2011, 115, 1403-1409.

(8) Leng, W.; Vikesland, P. J. Nanoclustered gold honeycombs for surface-enhanced Raman scattering. Analytical Chemistry 2013, 85, 1342-1349.

(9) Atwater, H. A.; Polman, A. Plasmonics for improved photovoltaic devices. Nature Materials 2010, 9, 205-213. 
(10) Jeseentharani, V.; Pugazhenthiran, N.; Mathew, A.; Chakraborty, I.; Baksi, A.; Ghosh, J.; Jash, M.; Anjusree, G. S.; Deepak, T. G.; Nair, A. S. et al. Atomically precise noble metal clusters harvest visible light to produce energy. ChemistrySelect 2017, 2, 1454-1463.

(11) Haberland, H. Looking from both sides. Nature 2013, 494, E1.

(12) Sinha-Roy, R.; García-González, P.; Weissker, H.-C.; Rabilloud, F.; FernándezDomínguez, A. I. Classical and ab initio plasmonics meet at sub-nanometric noble metal rods. ACS Photonics 2017, 4, 1484-1493.

(13) Hövel, H.; Fritz, S.; Hilgerand, A.; Kreibig, U.; Vollmer, M. Width of cluster plasmon resonances: bulk dielectric functions and chemical interface damping. Physical Review $B$ 1993, 48, 18178-18188.

(14) Campos, A.; Troc, N.; Cottancin, E.; Pellarin, M.; Weissker, H.-C.; Lermé, J.; Kociak, M.; Hillenkamp, M. Plasmonic quantum size effects in silver nanoparticles are dominated by interfaces and local environments. Nature Physics 2018, 15, 275-280.

(15) Tiggesbäumker, J.; Köller, L.; Meiwes-Broer, K.-H.; Liebsch, A. Blue shift of the Mie plasma frequency in Ag clusters and particles. Physical Review A 1993, 48, 1749-1752.

(16) Hilger, A.; Cüppers, N.; Tenfelde, M.; Kreibig, U. Surface and interface effects in the optical properties of silver nanoparticles. European Physical Journal D 2000, 10, 115118.

(17) Lünskens, T.; Heister, P.; Walenta, M. T. C. A.; Kartouzian, A.; Heiz, U. Plasmons in supported size-selected silver nanoclusters. Physical Chemistry Chemical Physics 2015, 17, $17541-17544$.

(18) Chakraborty, I.; Erusappan, J.; Govindarajan, A.; Sugi, K. S.; Udayabhaskararao, T.; 
Ghosh, A.; Pradeep, T. Emergence of metallicity in silver clusters in the 150 atom regime: a study of differently sized silver clusters. Nanoscale 2014, 6, 8024-8031.

(19) Yu, C.; Schira, R.; Brume, H.; von Issendorff, B.; Rabilloud, F.; Harbich, W. Optical properties of size selected neutral Ag clusters: electronic shell sructures and the surface plasmon resonance. Nanoscale 2018, 10, 20821-20827.

(20) Genzel, L.; Martin, T. P.; Kreibig, U. Dielectric function and plasma resonances of small metal particles. Zeitschrift für Physik B Condensed Matter 1975, 21, 339-346.

(21) Gaudry, M.; Lermé, J.; Cottancin, E.; Pellarin, M.; Vialle, J. L.; Broyer, M.; Prével, B.; Treilleux, M.; Mélinon, P. Optical properties of $\left(A u_{x} A g_{1-x}\right)_{n}$ clusters embedded in alumina: Evolution with size and stoichiometry. Physical Review B 2001, 64, 085407.

(22) Cottancin, E.; Celep, G.; Lermé, J.; Pellarin, M.; Huntzinger, J. R.; Vialle, J. L.; Broyer, M. Optical properties of noble metal clusters as a function of the size: comparison between experiments and a semi-quantal theory. Theoretical Chemistry Accounts 2006, 116, 514-523.

(23) Hillenkamp, M.; Domenicantonio, G. D.; O.Eugster,; Félix, C. Instability of Ag nanoparticles in $\mathrm{SiO}_{2}$ at ambient conditions. Nanotechnology 2006, 18, 015702.

(24) Lermé, J. Size evolution of the surface plasmon resonance damping in silver nanoparticles: confinement and dielectric effects. The Journal of Physical Chemistry $C$ 2011, $115,14098-14110$.

(25) Schmidt, M.; Masson, A.; Bréchignac, C. Oxygen and silver clusters: tansition from chemisorption to oxidation. Physical Review Letter 2003, 91, 243401.

(26) Schmidt, M.; Bréchignac, C. Silver and oxygen: Transition from clusters to nanoparticles. Comptes Rendus Physique 2016, 17, 481-484. 
(27) Wang, Y.; Gong, X.-G. First-principles study of small oxidized silver clusters. Journal of Nanoscience and Nanotechnology 2010, 10, 5500-5506.

(28) Klacar, S.; Hellman, A.; Panas, I.; Grönbeck, H. Oxidation of small silver clusters: a density functional theory study. The Journal of Physical Chemistry $C$ 2010, 114, $12610-12617$.

(29) Liao, M.-S.; Watts, J. D.; Huang, M.-J. Theoretical comparative study of oxygen adsorption on neutral and anionic $\mathrm{Ag}_{n}$ and $\mathrm{Au}_{n}$ clusters $(n=2-25)$. The Journal of Physical Chemistry C 2014, 118, 21911-21927.

(30) Bonačić-Koutecký, V.; Boiron, M.; Pittner, J.; Fantucci, P.; Koutecký, J. Structural and optical properties of small oxygen-doped- and pure-silver clusters. The European Physical Journal D 1999, 9, 183-187.

(31) Bürgel, C.; Mitrić, R.; Bonačić-Koutecký, V. Emissive properties of silver particles at silver oxide surface defects. Applied Physics A 2006, 82, 117-123.

(32) Casida, M. E. Recent advances in density functional methods; World Scientific, 1995; pp 155-192.

(33) Rabilloud, F. Assessment of the performance of long-range-corrected density functionals for calculating the absorption spectra of silver clusters. The Journal of Physical Chemistry A 2013, 117, 4267-4278.

(34) Kuisma, M.; Sakko, A.; Rossi, T.; Larsen, A. H.; Enkovaara, J.; Lehtovaara, L.; Rantala, T. T. Localized surface plasmon resonance in silver nanoparticles: Atomistic first-principles time-dependent density-functional theory calculations. Physical Review $B$ 2015, 91, 115431.

(35) Baseggio, O.; Vetta, M. D.; Fronzoni, G.; Stener, M.; Sementa, L.; Fortunelli, A.; Calzolar, A. Photoabsorption of icosahedral noble metal clusters: an efficient TDDFT 
approach to large-scale systems. Journal of Physical Chemistry C 2016, 120, 1277312782 .

(36) Schira, R.; Rabilloud, F. Localized surface plasmon resonance in free silver nanoclusters $\operatorname{Ag}_{n}, n=20-147$. The Journal of Physical Chemistry C 2019, 123, 6205-6212.

(37) Dreuw, A.; Head-Gordon, M. Failure of time-dependent density functional theory for long-range charge-transfer excited states: the zincbacteriochlorin-bacteriochlorin and bacteriochlorophyll-spheroidene complexes. Journal of the American Chemical Society 2004, 126, 4007-4016.

(38) Rabilloud, F. Description of plasmon-like band in silver clusters: The importance of the long-range Hartree-Fock exchange in time-dependent density-functional theory simulations. The Journal of Chemical Physics 2014, 141, 144302.

(39) Chai, J.-D.; Head-Gordon, M. Systematic optimization of long-range corrected hybrid density functionals. The Journal of Chemical Physics 2008, 128, 084106.

(40) Hay, P. J.; Wadt, W. R. Ab initio effective core potentials for molecular calculations. Potentials for $\mathrm{K}$ to Au including the outermost core orbitals. The Journal of Chemical Physics 1985, 82, 299-310.

(41) Schira, R.; Rabilloud, F. Effects of rare-gas matrices on the optical response of silver nanoclusters. The Journal of Physical Chemistry C 2018, 122, 27656-27661.

(42) Hariharan, P. C.; Pople, J. A. The influence of polarization functions on molecular orbital hydrogenation energies. Theoretica Chimica Acta 1973, 28, 213-222.

(43) Chen, M.; Dyer, J. E.; Li, K.; Dixon, D. A. Prediction of structures and atomization energies of small silver clusters, $\mathrm{Ag}_{n}, n<100$. The Journal of Physical Chemistry A 2013, 117, 8298-8313. 
(44) Harb, M.; Rabilloud, F.; Simon, D.; Rydlo, A.; Lecoultre, S.; Conus, F.; Rodrigues, V.; Félix, C. Optical absorption of small silver clusters: $A g_{n},(\mathrm{n}=4-22)$. The Journal of Chemical Physics 2008, 129, 194108.

(45) Frisch, M. J.; Trucks, G. W.; Schlegel, H. B.; Scuseria, G. E.; Robb, M. A.; Cheeseman, J. R.; Scalmani, G.; Barone, V.; Petersson, G. A.; Nakatsuji, H. et al. Gaussian 09 Revision D.01. 2009; Gaussian Inc. Wallingford CT.

(46) Allouche, A.-R. Gabedit-A graphical user interface for computational chemistry softwares. Journal of Computational Chemistry 2011, 32, 174-182.

(47) Reed, A. E.; Weinstock, R. B.; Weinhold, F. Natural population analysis. The Journal of Chemical Physics 1985, 83, 735-746.

(48) Lünskens, T.; Walenta, C. A.; Heister, P.; Kartouzian, A.; Heiz, U. Surface oxidation of supported, size-selected silver clusters. Journal of Cluster Science 2017, 28, 3185-3192.

(49) Douglas-Gallardo, O. A.; Soldano, G. J.; Mariscal, M. M.; Sanchez, C. G. Effects of oxidation on the plasmonic properties of aluminum nanoclusters. Nanoscale 2017, 9, $17471-17480$.

(50) de Heer, W. A. The physics of simple metal clusters: experimental aspects and simple models. Reviews of Modern Physic 1993, 65, 611-676. 